\title{
Novel nanoparticulate drug delivery systems
}

First draft submitted: 15 January 2016; Accepted for publication: 22 January 2016; Published online: 25 February 2016

Keywords: Alzheimer's disease $\bullet$ biomimetic nanoparticles $\bullet$ drug delivery nanosystems - neutrophil-mediated nanoparticle transport • photothermal cancer therapy

\section{Multifunctional GM1-modified} lipoprotein-like nanoparticles for the therapy of Alzheimer's Disease Evaluation of: Huang $\mathrm{M}, \mathrm{Hu} \mathrm{M}$, Song Q et al. GM1-modified lipoproteinlike nanoparticle: multifunctional nanoplatform for the combination therapy of Alzheimer's disease. ACS Nano. 9(11), 10801-10816 (2015).

Alzheimer's disease (AD) is the most common neurodegenerative disorder, affecting about $11 \%$ of people aged 65 years and older in the USA alone. According to the estimates, this number may nearly triple by 2050 [1] resulting in an increased health and economic burden, which urgently calls for more effective therapies.

The molecular basis for the formation of the extracellular amyloid plaques found in the brain of AD patients involves the strong neurotoxic effects of soluble amyloid- $\beta$ (A $\beta$ ) [2]. In their recent paper, Huang et al. have developed a flexible multifunctional noninvasive drug delivery system for accelerated $A \beta$ clearance, protection of neurons and inhibition of other pathological cascades induced by $A \beta$ accumulation.

Reconstituted high-density lipoprotein (rHDL) and monosialotetrahexosylganglioside (GM1)-rHDL nanoparticles were prepared by self-assembly from lipid-free apoE3 and 1,2-dimyristoyl-sn-glycero-3-phosphocholine (DMPC) liposomes or GM1-DMPC liposomes, respectively [3]. Surface plasmon resonance analysis showed a very high affinity of GM1-rHDL to monomeric and oligomeric $A \beta_{1-42}$, comparable with anti-A $\beta$ antibody binding. Following the extensive physicochemical analyses, in vivo experiments demonstrated a high level of GM1-rHDL in the brain after intranasal application in mice, confirming the feasibility of noninvasive administration of these nanoparticles.

Subsequent in vitro and in vivo experiments revealed a strong improvement of different $A \beta$ clearing pathways in the presence of GM1rHDL as compared with rHDL. In primary microglia, GM1-rHDL facilitated the cellular uptake and intracellular degradation of $A \beta_{1-42}$. Increased $A \beta$ degradation and an accelerated $A \beta$ brain-to-blood efflux after injection of ${ }^{125}$ I-labeled $A \beta_{1-42}$ oligomers into the unilateral hippocampus was also achieved in vivo with intranasally administered GM1-rHDL.

Beside increased $A \beta$ levels, $A D$ is characterized by loss of neurons and synapses leading to memory loss, cognitive deficits and ultimately to death. Consequently, a successful therapy should combine strategies for $A \beta$ clearance and neuroprotection. To verify the drugdelivery capacity of GM1-rHDL, the authors selected a neuroprotective octapeptide, $\mathrm{NAP}$, as a model drug for $\mathrm{AD}$. The resulting $\alpha$ NAP-GM1-rHDL particles increased the viability of $A \beta_{1-42}$ oligomer-treated primary neurons and reversed neuronal dysfunction by increasing the neurite length and branch point counts. Additionally, aNAP-GM1-rHDL
Ralf P Friedrich', Marina Pöttler ${ }^{1}$, Iwona Cicha', Stefan Lyer ${ }^{1}$, Christina Janko' \& Christoph Alexiou*,1 'ENT-Department, Section of Experimental Oncology \& Nanomedicine (SEON), Else Kröner-Fresenius-StiftungProfessorship for Nanomedicine, University Hospital Erlangen, Glueckstr. 10a, 91054 Erlangen, Germany *Author for correspondence: c.alexiou@web.de 
significantly decreased the glutamic acid-induced neurotoxicity, a process reported to be triggered by $\mathrm{A} \beta$ [4]. In $\mathrm{AD}$ mice, $\alpha$ NAP-GM1-rHDL were well-tolerated and reduced the $A \beta$ deposition, ameliorated the neurologic damage and alleviated the memory deficits.

In summary, the therapeutic benefits of these particles in terms of $A \beta$ clearance, neuroprotection and biosafety suggest their potential application for the treatment of AD.

\section{- Written by Ralf P Friedrich}

\section{Stimuli-responsive magnetic nanoparticles for photo-chemo-thermal cancer therapy Evaluation of: Yu J, Ju Y, Zhao L et al. Multistimuli-regulated photochemothermal cancer therapy remotely controlled via $\mathrm{Fe}_{5} \mathrm{C}_{2}$ nanoparticles. ACS Nano 10(1), 159-169 (2015).} A controlled drug delivery and release system would be of great advantage especially for cancer therapy. $\mathrm{Yu}$ et al. developed magnetic nanoparticles functionalized as drug carriers that can control drug delivery for combined cancer therapy. These multicontrolled drug carriers respond to two synergistic stimuli: on one hand, they are controlled by near-infrared (NIR) light, which is an attractive stimulus for drug release, because of its low phototoxicity and relatively deep tissue penetration compared with UV light. As the second stimulus $\mathrm{pH}$ is used, exploiting the $\mathrm{pH}$ differences between tumor and normal tissue. Additionally, NIR light generates heat enabling a photothermal therapy (PTT).

The authors employed carbon-coated iron carbide $\left(\mathrm{Fe}_{5} \mathrm{C}_{2}\right)$ nanoparticles, which are ideal as magnetic resonance imaging and light-absorbing agents, and can be actively guided by an external magnetic field. Such remote control allows their increased accumulation in the area of interest. Additionally, these nanoparticles were functionalized with bovine serum albumin for better water solubility and biocompatibility, and loaded with doxorubicin (DOX), a clinically used anticancer drug.

The in vitro studies demonstrated that upon cellular uptake of particles in lysosomes, DOX was released by a low $\mathrm{pH}$ and after NIR irradiation DOX was transferred to the nucleus. Moreover, NIR irradiation increased the local temperature and enhanced the permeability of the lysosomal membrane whereupon more of free DOX was released in the cytosol. In the ovarian tumor-bearing mice, site-specific targeting was achieved by placing a magnet above the tumor. Furthermore, upon a single intravenous application in the treated animals followed by irradiation with an $808 \mathrm{~nm}$ laser for $5 \mathrm{~min}$, synergistic effects of chemotherapy, PTT and magnetic drug targeting were demonstrated by the complete disappearance of the tumor within 27 days in the absence of systemic side effects.

Taken together, Yu et al. established a new multistimuli-triggered drug delivery system based on bovine serum albumin-functionalized $\mathrm{Fe}_{5} \mathrm{C}_{2}$ nanoparticles with improved DOX loading capacities. Following the cellular uptake, a controlled switch-on by $\mathrm{pH}$ and NIR allows photothermal heating, which results in increased drug release and directly causes cell death by PTT. Tumor targeting using the magnetic field amplifies specific drug delivery to the tumor site and increases therapeutic efficacy. Additionally, the delivery can be monitored by T2-weighted MRI. This type of multistimuli-controlled magnetic drug delivery combined with PTT has a great potential for cancer therapy.

- Written by Marina Pöttler \& Christoph Alexiou

\section{Platelet-mimicking nanoparticles for targeted drug delivery}

Evaluation of: Hu CM, Fang RH, Wang KC et al. Nanoparticle biointerfacing by platelet membrane cloaking. Nature 526(7571), 118-121 (2015).

The design of novel nanoparticles capable of delivering drugs to the diseased sites and organs is expected to improve the therapy of various disorders, for which the systemic therapies lack sufficient efficacy. Surface functionalization of such nanoparticles is proposed to extend their circulation time and to reduce the adverse reactions from the circulating immune cells [5]. In a recent study by $\mathrm{Hu}$ et al., biomimetic nanoparticles were developed by coating polymeric nanoparticles with the membranes of human platelets.

Platelet membrane vesicles, obtained from EDTAanticoagulated human blood from group 0 donors by plasma isolation and sonication, were fused with $100-\mathrm{nm}$ poly(lactic-co-glycolic acid) (PLGA) nanoparticles. These nanoconstructs, termed PNPs, had a diameter of $115 \mathrm{~nm}$ and showed consistent unilamellar membrane coatings over the polymeric cores in transmission electron microscopy. PNPs had a right-side-out membrane orientation as confirmed by immunogold staining and flow cytometric analysis of the intracellular or extracellular domain of CD47 [6]. Moreover, platelet membrane protein content $[7,8]$ including CD 47, CD55 and CD59, integrins $\alpha \mathrm{IIb}, \alpha 2, \alpha 5, \alpha 6, \beta 1$ and $\beta 3$, as well as GPIb $\alpha$, GPIV, GPV, GPVI, GPIX and CLEC-2 [7,8] was successfully transferred on the nanoparticles, whereas the prothrombotic molecules such as thrombin, ADP and thromboxane were absent. The PNPs were further examined for their immunocompatibility, showing a lack of complement activation, which indicates 
a reduced risk of pseudoallergic reaction commonly associated with injectable nanoformulations [9].

The platelet-like functionality of the particles was demonstrated by binding to human collagen IV in vitro, and to the damaged vasculature, in other words, subendothelial matrix of denuded human carotid artery ex vivo, and in a rat model of angioplasty-induced arterial damage. The therapeutic potential of PNPdirected docetaxel delivery to injured endothelium was validated in a rat model of coronary stenosis, whereby PNP-docetaxel treatment potently inhibited neointima formation and reduced the degree of stenosis. PNPs were also shown to accumulate on MRSA252 strain of methicillin-resistant Staphylococcus aureus, serving as an in vitro model of platelet-adhering pathogen. Used as a drug carrier for vancomycin, PNPs evoked a significant antimicrobial effect in vitro and substantially reduced the bacterial counts in a mouse model of systemic MRSA252 infection, as compared with free vancomycin.

Merging the nanomaterials technology, biochemistry and blood physiology this promising approach thus provides a novel and safer tool for developing disease-specific drug delivery nanocarriers.

\section{- Written by Iwona Cicha \& Stefan Lyer}

\section{Hijacking neutrophils for the transport of therapeutic nanoparticles across the blood vessel barrier \\ Evaluation of: Chu D, Gao J, Wang Z. Neutrophil-mediated delivery of therapeutic nanoparticles across blood vessel barrier for treatment of inflammation and infection. ACS Nano 9(12), 11800-11811 (2015).}

For therapeutic success, intravenously administered nano-pharmaceuticals must be able to reach their extravasal target tissues. Since endothelial monolayers of healthy blood vessels form a barrier for particles larger than $3 \mathrm{~nm}$ [10], alternative strategies are needed for effective transport of nanoparticles from blood into the tissues. In their article, Chu et al. show that migrating neutrophils can serve as carriers for certain nanoparticles from the bloodstream to the inflamed tissues. A proof-of-concept study in TNF- $\alpha$-induced scrotal inflammation demonstrated that adherent neutrophils internalize Cy5-labeled albumin nanoparticles $(130 \mathrm{~nm})$ via Fc $\gamma$ receptors and transport them through the blood vessel barrier to the cremaster muscle [11]. To confirm these findings, a mouse model of acute lung inflammation induced by intratracheal administration of lipopolysaccharide was applied, whereby activated neutrophils migrate from bloodstream to alveoli passing endothelial and epithelial barriers. After intravenous injection of albumin nanoparticles, the pool of nanoparticle-containing neutrophils decreased with time in blood and increased in bronchoalveolar fluid (BALF), indicating neutrophil transmigration. To rule out that lipopolysaccharide-induced endothelial permeability contributes to nanoparticle translocation, PEGcoated polystyrene nanoparticles $(130 \mathrm{~nm})$ which are not taken up by neutrophils were applied. Despite much longer circulation of PEG nanoparticles in the blood as compared with the albumin nanoparticles, no PEG nanoparticles were detected in BALF or in transmigrated neutrophils, indicating that the transport of albumin nanoparticles across blood vessel in lung inflammation is an active process mediated by neutrophils rather than the passive nanoparticle diffusion through the vasculature. Supporting this conclusion, albumin nanoparticle transport was completely abolished after neutrophil depletion using antimouse $\mathrm{Gr}-1$ antibody.

Neutrophil-mediated nanoparticle transport was subsequently implemented for the therapy of acute lung injury, which is accompanied by NF- $\mathrm{kB}$-mediated cytokine release by lung macrophages. Compared to the free drug, $20 \mathrm{~h}$ post intravenous injection of albumin nanoparticles loaded with the NF- $\mathrm{BB}$ inhibitor TPCA-1, increased concentrations of TCPA-1 were detected in BALF, resulting in reduced leukocyte infiltration, lung permeability and cytokine release. Furthermore, albumin nanoparticles loaded with broad-spectrum antibiotic (cefoperazone acid) were used to treat Pseudomonas aeruginosa-mediated acute pneumonia in mice. These nanoparticles lead to threefold reduction in the number of colony-forming units in BALF compared with the free drug.

Taken together, the presented results confirmed the capacity of neutrophils as carriers of therapeutic albumin nanoparticles across the blood vessel barrier into inflamed tissues. Since human neutrophils represent $50-70 \%$ of all circulating leukocytes [12], this drug delivery platform might have a significant impact on the treatment of inflammatory diseases and infections in the future.

\section{- Written by Christina Janko}

\section{Financial \& competing interests disclosure}

The authors have no relevant affiliations or financial involvement with any organization or entity with a financial interest in or financial conflict with the subject matter or materials discussed in the manuscript. This includes employment, consultancies, honoraria, stock ownership or options, expert testimony, grants or patents received or pending, or royalties.

No writing assistance was utilized in the production of this manuscript. 


\section{References}

1 Alzheimer's Association. Alzheimer's disease facts and figures. Alzheimers Dement. 10(2), e47-92 (2014).

2 Bloom GS. Amyloid-beta and tau: the trigger and bullet in Alzheimer disease pathogenesis. JAMA Neurol. 71(4), 505-508 (2014).

3 Song Q, Huang M, Yao L et al. Lipoprotein-based nanoparticles rescue the memory loss of mice with Alzheimer's disease by accelerating the clearance of amyloidbeta. ACS Nano 8(3), 2345-2359 (2014).

4 Talantova M, Sanz-Blasco S, Zhang X et al. Abeta induces astrocytic glutamate release, extrasynaptic NMDA receptor activation, and synaptic loss. Proc. Natl Acad. Sci. USA 110(27), E2518-E2527 (2013).

5 Anselmo AC et al. Platelet-like nanoparticles: mimicking shape, flexibility, and surface biology of platelets to target vascular injuries. ACS Nano 8(11), 11243-11253 (2014).

6 Olsson M, Bruhns P, Frazier WA, Ravetch JV, Oldenborg PA. Platelet homeostasis is regulated by platelet expression of CD47 under normal conditions and in passive immune thrombocytopenia. Blood 105(9), 3577-3582 (2005).

7 Rivera J, Lozano ML, Navarro-Núñez L, Vicente V. Platelet receptors and signaling in the dynamics of thrombus formation. Haematologica 94(5), 700-711 (2009).

8 Andrews RK, Berndt MC. Platelet physiology and thrombosis. Thromb. Res. 114 (5-6), 447-453 (2004).

9 Szebeni J. Complement activation-related pseudoallergy: a stress reaction in blood triggered by nanomedicines and biologicals. Mol. Immunol. 61(2), 163-173 (2014).

10 Mehta D, Malik AB. Signaling mechanisms regulating endothelial permeability. Physiol. Rev. 86(1), 279-367 (2006).

11 Wang Z, Li J, Cho J, Malik AB. Prevention of vascular inflammation by nanoparticle targeting of adherent neutrophils. Nat. Nanotechnol. 9(3), 204-210 (2014).

12 Mayadas TN, Cullere X, Lowell CA. The multifaceted functions of neutrophils. Annu. Rev. Pathol. 9, 181-218 (2014). 